\title{
Natural enemy or friend? Pneumonia in the very elderly critically ill patient
}

\author{
Michael S. Niederman
}

Affiliation: Pulmonary and Critical Care Medicine, New York Presbyterian/Weill Cornell Medical Center, New York, NY, USA.

Correspondence: Michael S. Niederman, Cornell University Joan and Sanford I Weill Medical College, Pulmonary and Critical Care Medicine, 425 East 61st St, 4th Floor, New York, NY 10065, USA. E-mail: msn9004amed.cornell.edu

@ERSpublications

Pneumonia is a common problem in the elderly with higher short- and long-term mortality than younger patients and similar age patients with other illnesses. Aggressive care may not be justified and broad-spectrum antibiotics may create antibiotic resistant pathogens that harm others. http://bit.ly/2P8LUNp

Cite this article as: Niederman MS. Natural enemy or friend? Pneumonia in the very elderly critically ill patient. Eur Respir Rev 2020; 29: 200031 [https://doi.org/10.1183/16000617.0031-2020].

Pneumonia has been a serious cause of morbidity and mortality for many years and, more than 100 years ago, Sir William Osler referred to it as "the natural enemy of the old man" [1]. Many studies have documented that elderly individuals have a higher frequency of illness, increased mortality and more subtle clinical features compared to younger populations. This too was known by Osler who commented that "it is not improbable that debility lowers the vitality and renders the individual susceptible" and when the illness occurs, it can be "without chill; the cough and expectoration are slight" and the physical findings non-specific [1]. He noted that while fever was not usually prominent, patients often had altered mental status and that pneumonia could precipitate congestive heart failure [1].

In much of the literature on this topic, elderly has been defined as $>65$ years of age, and the identification of subgroups, based on age, in this population has not always been part of large series. In the current issue of the European Respiratory Review, CILlónIZ et al. [2] have focused on the problem of pneumonia in the "very elderly", a specific subgroup defined as those aged $>85$ years, and have specifically discussed those with critical illness. This is a very important and controversial topic, raising issues about whether age itself is important in defining the risk and prognosis of pneumonia, and which very elderly patients with severe illness should be managed in an intensive care unit (ICU), and in which patients the focus should be on comfort, not heroic life support.

Many studies have suggested that in the elderly, pneumonia can start a cascade of events that results in mortality, even after the patient has left the hospital. KAPLAN et al. [3] studied 158960 hospitalised patients with community-acquired pneumonia (CAP) aged >65 years and compared them to 794333 matched control patients in the same age range, but admitted to hospital for other diseases. The in-hospital mortality rate for CAP was $11 \%$, but those who were discharged had an additional mortality in the next year of 33.6\%. Thus, for many patients, pneumonia is the start of an inexorable decline. The short term and 1-year mortality rate for patients admitted with other diagnoses was not as high as with pneumonia.

The very elderly have a higher mortality from pneumonia than other age groups. LunA et al. [4] observed that age $>80$ years was a risk factor for mortality (OR 2.0) and that in this group, mortality for those with

Provenance: Commissioned article, peer reviewed.

Received: 01 Feb 2020 | Accepted: 12 Feb 2020

Copyright $\odot$ ERS 2020. This article is open access and distributed under the terms of the Creative Commons Attribution Non-Commercial Licence 4.0. 
$>1$ comorbidity was higher than for those with $\leqslant 1$ comorbidities. These data suggest that above 80 years of age there is not "healthy aging", since in this group pneumonia mortality is greater, even in those with one comorbidity than in younger populations with multiple comorbidities. In contrast, LE BorGNE et al. [5] observed that patients aged $>90$ years who were admitted to the ICU with all diagnoses (including $52 \%$ with respiratory failure) had a mortality rate of $42.6 \%$, although age was not a predictor of death, but rather other factors such as mechanical ventilation, need for dialysis and the withholding or withdrawing of life support were most predictive.

The worse outcomes of pneumonia that are seen in the very elderly are likely the result of multiple factors. These include immune function decline, a high rate of serious comorbidity, high rates of frailty, impaired functional status, and delays in pneumonia diagnosis. Delays in recognising pneumonia can be the result of non-classical or subtle symptoms that do not lead to a consideration of a pneumonia diagnosis. Chest radiographs can be negative early in the course of pneumonia, especially in older patients, and this also adds to diagnostic delay which in turn can lead to therapy later in the course of illness, adding to mortality [6]. In one study of 96 elderly patients with CAP (mean age 83 years), the chest radiograph on presentation was positive in only $47 \%$, and was a particularly unreliable tool in patients with advanced degrees of frailty [7]. The chest radiograph had a negative predictive value of $73 \%$ in those with low degrees of frailty but only $53 \%$ in those with advanced levels of frailty.

The site of care decision for CAP patients is important but one that is immeasurably harder in the elderly. In many settings, prognostic scoring indices are used to guide this decision, but in the elderly, prognostic scoring is less accurate than in younger populations, and for any given score the very elderly have a higher mortality [4]. However, most prognostic scoring tools heavily weight age as a risk factor, and thus for similar severity of illness the elderly get a higher score, a predicted higher mortality, and an apparent higher need for advanced care such as ICU [8]. Thus, the elderly have higher predicted mortality than younger patients for the same degree of illness, and higher observed mortality than younger populations in any given prognostic scoring category. Thus, it is hard to decide which of these patients should be considered for ICU admission, and to what extent the ICU can be beneficial for the very elderly, compared to younger patients.

The review by Cillóniz et al. [2] raises an additional question in the site of care decision, and that is whether the very elderly with critical illness should always be admitted to an ICU, or if for some this would not be appropriate, given the patients' wishes, their short- and long-term prognosis, and the likelihood of benefit from ICU admission. As an alternative, they present the use of intermediate care units which have been reported to reduce overall hospital mortality, compared to hospitals without these units. This may be because these units provide an appropriate level of care for many patients, and do not force a dichotomous choice between ICU or no ICU care, the latter offering little chance of recovery for some patients. Decisions about care at the end of life in the elderly are complex, but should be influenced by a realistic view of whether for each patient we are prolonging life or suffering. For some patients, ICU admission offers little chance of recovery or long-term survival. As discussed above, pneumonia may be the "beginning of the end" for some patients, with both high short- and long-term mortality, and ICU care may not be appropriate for them. Osler understood this when he changed his view about pneumonia to an illness that was the "friend of the aged" [1]. Of course, the irony of this description is that at age 70, Osler developed pneumonia with a Haemophilus influenzae empyema and died.

One other important issue in pneumonia treatment is the use of antibiotics. The elderly often have more drug resistant bacteria than younger patients, particularly those who have multiple comorbidities, live in chronic care facilities and have received multiple courses of antibiotics. In this population, empiric therapy is usually with a broad-spectrum agent. Antibiotics are among the most used therapies in dying patients, in and out of the ICU, and doctors often use them even when they will not provide CPR. In one study, $45 \%$ of patients got antibiotics in the last $24 \mathrm{~h}$ of life, independent of a do not resuscitate order, and antibiotics were the most common intervention after hydration [9]. In another study of 95 ICU patients with no planned escalation of care, antibiotics were discontinued less often than dialysis and pressors [10]. Among 423 ICU patients in Toronto (Canada) and Jerusalem (Israel), the absence of a limitation of care order was the only independent predictor of the emergence of antibiotic resistance [11]. However, one needs to consider that the use of our best antibiotics may have limited benefit to patients who develop repeated infections and are not likely to recover because of the severity of their chronic and acute illnesses. When patients receive antibiotics, with little likelihood of benefit, but at the same time therapy induces the emergence of even more antibiotic resistant pathogens (which can occur in up to $25 \%$ of these patients), it may result in harm to other patients in the hospital and ICU [12]. Highly resistant pathogens can spread in the ICU to other, more healthy individuals and infect them with organisms that cannot be effectively treated, and can result in the death of these other patients. If this happens, one could question whether the use of broad-spectrum antibiotics in the very elderly critically ill patient, with a low chance of benefitting, 
created more harm than good. For this population, we may need to consider Osler's view that pneumonia can be the friend of the elderly, and focus our efforts on patient comfort and relief of suffering, rather than treatment with a limited chance of individual patient benefit and a risk of harm to others in the ICU.

Conflict of interest: M.S. Niederman reports personal fees from Bayer, Shionogi, Polyphor, Pfizer and Nabriva, and grants from Bayer and Merck, outside the submitted work.

\section{References}

1 Berk SL. Bacterial pneumonia in the elderly: the observations of Sir William Osler in retrospect. J Am Geriatr Soc 1984; 32: 683-685.

2 Cillóniz C, Dominedò C, Pericàs JM, et al. Community-acquired pneumonia in critically ill very old patients: a growing problem. Eur Respir Rev 2020; 29: 190126.

3 Kaplan V, Clermont G, Griffin MF, et al. Pneumonia: still the old man's friend? Arch Intern Med 2003; 163: 317-323.

4 Luna CM, Palma I, Niederman MS, et al. The impact of age and comorbidities on the mortality of patients of different age groups admitted with community-acquired pneumonia. Ann Am Thorac Soc 2016; 13: 1519-1526.

5 Le Borgne P, Maestraggi Q, Couraud S, et al. Critically ill elderly patients ( $\geqslant 90$ years): clinical characteristics, outcome and financial implications. PLoS One 2018; 13: e0198360.

6 Niederman MS. Imaging for the management of community-acquired pneumonia: what to do if the chest radiograph is clear. Chest 2018; 153: 583-585.

7 Ticinesi A, Lauretani F, Nouvenne A, et al. Lung ultrasound and chest $\mathrm{x}$-ray for detecting pneumonia in an acute geriatric ward. Medicine 2016; 95: e4153.

8 Brito V, Niederman MS. Predicting mortality in the elderly with community-acquired pneumonia: should we design a new car or set a new 'speed limit'? Thorax 2010; 65: 944-945.

9 Phua J, Kee AC, Tan A, et al. End-of-life care in the general wards of a Singaporean hospital: an Asian perspective. J Palliat Med 2011; 14: 1296-1230.

10 Morgan CK, Varas GM, Pedroza C, et al. Defining the practice of "no escalation of care" in the ICU. Crit Care Med 2014; 42: 357-361.

11 Levin PD, Simor AE, Moses AE, et al. End-of-life treatment and bacterial antibiotic resistance: a potential association. Chest 2010; 138: 588-594.

12 Niederman MS, Berger JT. The delivery of futile care is harmful to other patients. Crit Care Med 2010; 38: Suppl. 10, S518-S522. 